\title{
Association study between a polymorphic poly-T repeat sequence in the promoter of the somatostatin gene and metabolic syndrome
}

\author{
Monique Tremblay, Diane Brisson and Daniel Gaudet * (D)
}

\begin{abstract}
Background: Metabolic syndrome is a cluster of factors associated with an increased risk of developing type 2 diabetes mellitus (T2D) and coronary artery disease (CAD). It is a complex disorder resulting from the interaction between various environmental factors and genetic susceptibility. The somatostatin (SST) gene has been shown to regulate a wide range of functions, particularly in energy homeostasis. In addition, low levels of SST have been reported to have effects on the progression of metabolic syndrome components. The aim of this study was therefore to evaluate the association between polymorphic T sequences in the promoter of the SST gene and metabolic syndrome expression.
\end{abstract}

Methods: We studied 1725 French-Canadian subjects from a founder population selected on the basis of having a positive family history of dyslipidemia, CAD or T2D. The analysis were performed on four groups created according to the poly T polymorphism length in the $5^{\prime}$ flanking promoter region of SST. Anova, Ancova and logistic regression models and Chi 2 analyses were used to evaluate the association between the poly $T$ polymorphisms and metabolic syndrome components expression.

Results: Analyses showed that means, frequencies and odds ratio of metabolic syndrome components expression increase as the number of poly-T repeats in the promoter region of SST increases. Women exhibit more significant differences than men. However, the trends are the same in both genders and differences for most of the components are significant in the entire sample.

Conclusion: Those results suggest that the poly T polymorphisms in the SST promoter region may influence several metabolic processes implicated in metabolic syndrome expression. More analyses are needed to document the mechanisms that could underlie genetic regulation effect of SST on metabolic syndrome components and to clarify its specific role.

Keywords: Somatostatin, Poly-T repeat, Gene polymorphism, Metabolic syndrome

\footnotetext{
* Correspondence: daniel.gaudet@umontreal.ca

Lipidology Unit, Community Genomic Medicine Center, Department of

Medicine, Université de Montréal and ECOGENE-21 Clinical and Translational

Research Center, 225 St-Vallier Chicoutimi, Québec G7H 7P2, Canada
}

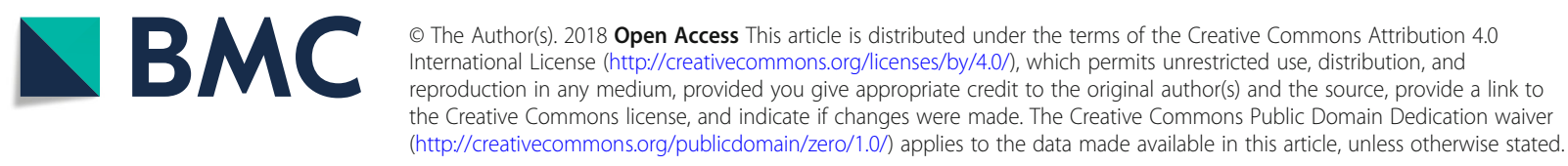




\section{Background}

Metabolic syndrome is a cluster of interrelated common clinical disorders occurring together more often than by chance alone. It includes hyperglycaemia, hypertension, elevated triglyceride (TG) levels, low high-density lipoprotein (HDL) cholesterol levels and central obesity. The presence of 3 out of 5 risk factors indicates the presence of metabolic syndrome [1]. Metabolic syndrome has received worldwide attention for the past several years because of its increasing prevalence, which now ranges from $20 \%$ to nearly $35 \%$ of the adult population in almost all Western countries and, increasingly, in young age groups [2]. It has been estimated that people with metabolic syndrome are at twice the risk of developing coronary artery disease (CAD), compared with those without the syndrome, and experience a five-fold increased risk of type 2 diabetes (T2D) [1].

Numerous genome-wide association studies (GWAS) have been carried out for many common diseases, including metabolic syndrome and related covariables [3, 4]. More than 2000 robust association studies were published only between 2005 and 2012 [5]. Unfortunately, most of the gene variants identified usually explain only a small fraction of the heritability of these diseases, so that the value of such analyses is often met by scepticism. However, GWAS are not solely useful for identifying risk factors. Combined with functional studies and gene-environment interaction analyses, the mapping of gene networks associated with complex diseases, such as metabolic syndrome, may be essential to unveiling their aetiology and biological mechanisms [4].

Previous linkage analyses and association studies led us to target the somatostatin (SST) gene as a potential candidate with a significant position in the metabolic syndrome-associated gene network. We previously found a poly- $\mathrm{T}$ repeat sequence (rs34872250) in its promoter and documented an association between the length of the repeat and increased risk of hypertension, especially among women and overweight/obese individuals [6].

SST is a ubiquitous peptide that exerts effective inhibition of growth hormone $(\mathrm{GH})$ release. It influences somatic growth and body weight by regulating the intestinal absorption of nutrients, gastro-intestinal motility, food intake and energy homeostasis. It also inhibits the production of insulin and glucagon, and limits the absorption of carbohydrates and TG in the small intestine. SST analogue has been used as therapy in the management of obesity and T2D. SST also inhibits a variety of other physiological functions in the central and peripheral nervous system, in the gastrointestinal tract and in the pancreas. Among its various activities, SST acts as a neurohormone and a neurotransmitter, and exercises autocrine and paracrine functions [7-9].
Considering the association between SST and numerous pathways well known to be involved in metabolic syndrome aetiology, as well as the central place of hypertension and overweight/obesity in its expression, it appears indispensable to investigate the association between poly- $\mathrm{T}$ repeat polymorphism and metabolic syndrome expression.

The aim of this study was therefore to investigate the relationship between the poly- $\mathrm{T}$ repeat polymorphism in the promoter of the SST gene and the expression of metabolic syndrome components.

\section{Methods}

\section{Subjects and clinical data}

This study comprised a sample of 1725 French-Canadian subjects from the Saguenay-Lac-Saint-Jean region of Quebec (Canada), selected on the basis of having a positive family history of dyslipidemia or CAD. All subjects had agreed to participate in studies on genetic determinants of T2D and/or CAD combining GWAS and candidate gene strategies $[10,11]$. To be included in the analyses, all subjects had to be carriers of one of the four SST poly-T genotypes (13/13,13/14, 13/15 and 13/16), as previously described [6].

The presence of 3 or more of the following components was used for the diagnosis of metabolic syndrome: waist circumference $>102 \mathrm{~cm}$ in men or $>88 \mathrm{~cm}$ in women (abdominal obesity); TG $\geq 1.7 \mathrm{mmol} / \mathrm{L}$ (hypertriglyceridemia); HDL-cholesterol $<1.03 \mathrm{mmol} / \mathrm{L}$ in men or $<1.3 \mathrm{mmol} / \mathrm{L}$ in women (low HDL-cholesterol levels); blood pressure $\geq 130 \mathrm{mmHg}$ for systolic blood pressure or $\geq 85 \mathrm{mmHg}$ for diastolic blood pressure or hypertensive treatment (hypertension); fasting glucose > $5.6 \mathrm{mmol} / \mathrm{L}$, or drug treatment for elevated glucose (hyperglycaemia) [1]. T2D was defined according to the World Health Organization criteria as a 2-h glucose concentration $>11.1 \mathrm{mmol} / \mathrm{L}$ following a $75 \mathrm{~g}$ oral glucose load, whereas a normal glucose tolerance state was characterized as a 2-h glucose concentration below $7.8 \mathrm{mmol} / \mathrm{L}$. Subjects with fasting TG $>20 \mathrm{mmol} / \mathrm{L}$ or BMI $>40 \mathrm{~kg} / \mathrm{m}^{2}$, those taking drugs known to affect blood lipid levels and those known for abusive alcohol consumption were excluded. Body weight, height, and waist girth were determined according to the procedures of the Airlie conference [12]. Subjects gave their informed consent to participate in this study and were assigned a code that systematically de-identifies all clinical data [13]. This project received the approval of the Chicoutimi Hospital Ethics Committee and was conducted in accordance with the Declaration of Helsinki.

\section{Biochemical analysis}

Blood samples were obtained after a 12-h overnight fast from the cephalic vein into vacutainer tubes containing 
EDTA. The HDL subfraction was obtained after precipitation of LDL $(\mathrm{d}>1.006 \mathrm{~g} / \mathrm{ml})$ in the infranatant with heparin and $\mathrm{MnCl}_{2}$ [14]. Plasma glycerol concentrations were measured with a Technicon RA-500 analyzer (Bayer Corporation), and enzymatic reagents were obtained from Randox (Randox Laboratories). Glucose, free fatty acid and TG levels were measured with enzymatic assays on a CX7 analyzer (Beckman, Fullerton, CA, USA) [15]. Plasma LDL cholesterol levels were estimated using the Friedewald formula [16]. When TG levels were higher than $4.5 \mathrm{mmol} / \mathrm{L}$, plasma LDL-cholesterol levels were calculated using a validated method [17].

\section{Microsatellite analysis}

The length of the poly- $T$ sequence was determined by DNA sequencing, using the BigDye ${ }^{\mathrm{Tw}}$ terminator kit (Applied Biosystems Instruments, ABI, Foster City, CA, USA). The size standard was produced by PCR amplification of each individual clone identified with a fluorescent marker. Sample electrophoresis and data analysis PCR products were resolved and detected by capillary electrophoresis, using an ABI PRISM 3100 Genetic Analyzer, a multi-colour fluorescence-based DNA analysis system with 16 capillaries operating in parallel, with denaturing polymer POP4 (Perkin-Elmer, Shelton, CT, USA). Fragment sizing was supported using the Genes$\operatorname{can}^{\mathrm{Tm}}$ (ABI, Foster Citym CA, USA).

\section{Statistical analysis}

Continuous variables were compared using analyses of variance (ANOVA) followed by the Bonferroni post hoc test and analyses of covariance (ANCOVA) including age and gender as covariates, or the non-parametric Kruskal-Wallis and Mann-Whitney U tests. Variables with skewed distribution were $\log _{10}$-transformed before analyses and geometrical means are presented. Chi square $\left(\chi^{2}\right)$ statistics were used to analyze distributions of categorical variables. Binary logistic regression models were constructed in order to calculate the relative odds of exhibiting components of metabolic syndrome according to the SST poly-T polymorphism genotype. The $13 / 13$ genotype was considered as the reference group to which an odds ratio (OR) of one was set for comparison purposes. P-values were two-sided. All statistical analyses were performed with the SPSS package (releases 11.5 or 21 , SPSS, Chicago Ill, USA).

\section{Results}

The subjects' characteristics are shown in Table 1. In the entire sample, significant differences $(p<0.01)$ were observed between the 13/13 and 13/16 genotypes for TG levels, waist girth, BMI and systolic blood pressure. There were also significant differences in systolic blood pressure levels between 13/14 and 13/16, and between 13/13 and
13/15 subjects. The tendency of TG levels to increase with the rising number of poly-T repeats is also interesting to note. Slightly different results were observed according to gender. In women, differences in BMI, systolic and diastolic blood pressure between genotypes are significant and they follow the same trend for waist girth. In men, only differences in TG and HDL-cholesterol concentrations are significant. Results remain the same after the inclusion of age and gender as a covariate.

As shown in Table 2, the prevalence of abdominal obesity, hypertriglyceridemia, hypertension and metabolic syndrome are significantly different $(p<0.05)$ across SST poly-T repeat groups in the entire sample, whereas there was a tendency toward low HDL-cholesterol levels and hyperglycemia. In women, significant differences in the prevalence of hypertension and metabolic syndrome diagnosis were noted, and there was a tendency to develop abdominal obesity. In men, only hypertriglyceridemia prevalence was significantly different across the groups.

Table 3 shows various multivariate models, including age and gender as covariates, in which each components as well as the metabolic syndrome are one after the other the dependent variable. Obtained results confirmed the increased odds of exhibiting metabolic syndrome components associated with the $13 / 16$ poly- $T$ repeat genotype when compared with the $13 / 13$ poly- $\mathrm{T}$ repeat genotype. In the entire sample, the risk was significantly increased for all metabolic syndrome components among subjects with the 13/16 genotype. In addition, the risks of hypertriglyceridemia and hypertension also increased significantly among the 13/15 carriers. Differences appear between genders, with the risk of hypertriglyceridemia and low HDL-cholesterol level being significant in men, while the risk of abdominal obesity, hypertension, hyperglycemia and metabolic syndrome are significant in women.

\section{Discussion}

Our study shows that the length of the SST poly-T repeat polymorphism is significantly associated with the expression of metabolic syndrome components, as well as the metabolic syndrome diagnosis itself. Although effects of SST analogue administration on metabolic syndrome component expression have been previously studied, [9] to the best of our knowledge, this is the first study on the association between the SST genotype and metabolic syndrome.

The SST gene is located on chromosome 3q27, a quantitative trait loci (QTL) associated with multiple representative traits of metabolic syndrome. Several familial and population studies have confirmed the existence of links between this QTL and metabolic syndrome. A number of candidate genes associated with the expression of the 
Table 1 Subjects' characteristics according to the SST poly T repeat polymorphism genotype in the entire sample and according to gender

\begin{tabular}{|c|c|c|c|c|c|c|c|c|c|c|c|c|c|c|}
\hline \multirow{2}{*}{$\begin{array}{l}\text { All subjects } \\
\text { Genotype } \\
\mathrm{n}\end{array}$} & \multicolumn{3}{|l|}{$13 / 13$} & \multicolumn{3}{|l|}{$13 / 14$} & \multicolumn{3}{|l|}{$13 / 15$} & \multicolumn{3}{|l|}{$13 / 16$} & \multirow[t]{2}{*}{$p$} & \multirow[t]{2}{*}{$p^{*}$} \\
\hline & \multicolumn{3}{|l|}{913} & \multicolumn{3}{|l|}{69} & \multicolumn{3}{|l|}{571} & \multicolumn{3}{|l|}{172} & & \\
\hline Gender M/W \% & \multicolumn{3}{|c|}{$52.0 / 48.0$} & \multicolumn{3}{|c|}{$55.1 / 44.9$} & \multicolumn{3}{|c|}{$50.6 / 49.4$} & \multicolumn{3}{|c|}{$57.6 / 42.4$} & \multicolumn{2}{|l|}{0.42} \\
\hline Age (years) & 49.7 & \pm & 11.8 & 47.4 & \pm & 11.7 & 50.1 & \pm & 12.2 & 50.3 & \pm & 12.1 & 0.33 & \\
\hline $\mathrm{CT}(\mathrm{mmol} / \mathrm{l})$ & 5.96 & \pm & 1.60 & 6.01 & \pm & 1.85 & 6.00 & \pm & 1.68 & 6.33 & \pm & $2.01 a$ & 0.07 & 0.06 \\
\hline $\mathrm{HDL}-\mathrm{C}(\mathrm{mmol} / \mathrm{l})$ & 1.20 & \pm & 0.45 & 1.14 & \pm & 0.41 & 1.20 & \pm & 0.47 & 1.11 & \pm & 0.44 & 0.07 & 0.17 \\
\hline $\mathrm{LDL}(\mathrm{mmol} / \mathrm{l})$ & 3.66 & \pm & 1.47 & 3.70 & \pm & 1.33 & 3.59 & \pm & 1.25 & 3.74 & \pm & 1.62 & 0.63 & 0.71 \\
\hline $\mathrm{TG}(\mathrm{mmol} / \mathrm{l})^{\mathrm{a}}$ & 1.80 & \pm & 2.22 & 1.89 & \pm & 2.26 & 1.90 & \pm & 2.12 & 2.19 & \pm & $3.11 \mathrm{a}$ & 0.007 & 0.01 \\
\hline WG (cm) & 90.1 & \pm & 13.1 & 92.3 & \pm & 13.5 & 90.6 & \pm & 13.0 & 94.0 & \pm & $13.6 \mathrm{ac}$ & 0.004 & 0.009 \\
\hline $\mathrm{BMI}\left(\mathrm{kg} / \mathrm{m}^{2}\right)$ & 26.8 & \pm & 4.5 & 28.0 & \pm & 5.0 & 27.0 & \pm & 4.5 & 28.0 & \pm & $4.5 \mathrm{ac}$ & 0.002 & 0.003 \\
\hline Glycaemia $(\mathrm{mmol} / \mathrm{l})^{a}$ & 5.58 & \pm & 1.49 & 5.87 & \pm & 1.75 & 5.67 & \pm & 1.68 & 5.82 & \pm & 1.56 & 0.06 & 0.05 \\
\hline Systolic BP (mmHg) & 124.9 & \pm & 19.4 & 122.2 & \pm & 15.9 & 128.5 & \pm & 20.5 & 131.1 & \pm & $22.5 \mathrm{ab}$ & 0.001 & $<0.001$ \\
\hline Diastolic BP (mmHg) & 78.4 & \pm & 11.5 & 79.0 & \pm & 10.9 & 79.2 & \pm & 11.1 & 80.9 & \pm & $11.4 \mathrm{a}$ & 0.05 & 0.08 \\
\hline $\mathrm{FFA}(\mathrm{mmol} / \mathrm{l})^{\mathrm{a}}$ & 0.49 & \pm & 0.25 & 0.45 & \pm & 0.24 & 0.50 & \pm & 0.33 & 0.53 & \pm & 0.28 & 0.14 & 0.12 \\
\hline Glycerol $(\mathrm{mmol} / \mathrm{l})^{\text {a }}$ & 0.07 & \pm & 0.09 & 0.06 & \pm & 0.03 & 0.07 & \pm & 0.28 & 0.07 & \pm & 0.06 & 0.52 & 0.28 \\
\hline \multicolumn{15}{|l|}{ Men } \\
\hline Genotype & $13 / 13$ & & & $13 / 14$ & & & $13 / 15$ & & & $13 / 16$ & & & $\mathrm{p}$ & $p^{* *}$ \\
\hline$n$ & 475 & & & 38 & & & 289 & & & 99 & & & & \\
\hline Age (years) & 49.4 & \pm & 11.2 & 48.2 & \pm & 11.2 & 48.8 & \pm & 11.7 & 49.9 & \pm & 11.4 & 0.74 & \\
\hline $\mathrm{CT}(\mathrm{mmol} / \mathrm{l})$ & 5.93 & \pm & 1.57 & 6.17 & \pm & 1.43 & 5.98 & \pm & 1.61 & 6.35 & \pm & 2.14 & 0.12 & 0.106 \\
\hline $\mathrm{HDL}-\mathrm{C}(\mathrm{mmol} / \mathrm{l})$ & 1.04 & \pm & 0.34 & 1.01 & \pm & 0.33 & 1.02 & \pm & 0.31 & 0.94 & \pm & $0.28 \mathrm{a}$ & 0.036 & 0.034 \\
\hline $\mathrm{LDL}(\mathrm{mmol} / \mathrm{l})$ & 3.73 & \pm & 1.46 & 4.03 & \pm & 1.50 & 3.73 & \pm & 1.30 & 3.79 & \pm & 1.71 & 0.67 & 0.68 \\
\hline $\mathrm{TG}(\mathrm{mmol} / \mathrm{l})^{\mathrm{a}}$ & 1.90 & \pm & 2.30 & 2.08 & \pm & 2.29 & 2.00 & \pm & 2.12 & 2.44 & \pm & $3.61 \mathrm{a}$ & 0.009 & 0.008 \\
\hline WG (cm) & 96.3 & \pm & 10.4 & 96.8 & \pm & 11.4 & 96.1 & \pm & 11.0 & 99.1 & \pm & 11.5 & 0.09 & 0.10 \\
\hline $\mathrm{BMI}\left(\mathrm{kg} / \mathrm{m}^{2}\right)$ & 27.4 & \pm & 3.9 & 28.1 & \pm & 4.2 & 27.4 & \pm & 4.0 & 28.2 & \pm & 4.1 & 0.23 & 0.22 \\
\hline Glycaemia $(\mathrm{mmol} / \mathrm{l})^{a}$ & 5.77 & \pm & 1.64 & 6.31 & \pm & 2.12 & 5.74 & \pm & 1.48 & 5.87 & \pm & 1.31 & 0.11 & 0.09 \\
\hline Systolic BP (mmHg) & 125.6 & \pm & 17.0 & 121.9 & \pm & 17.9 & 127.3 & \pm & 18.9 & 127.7 & \pm & 18.5 & 0.17 & 0.19 \\
\hline Diastolic BP (mmHg) & 80.1 & \pm & 11.1 & 79.2 & \pm & 11.3 & 79.4 & \pm & 10.4 & 80.6 & \pm & 10.9 & 0.73 & 0.78 \\
\hline $\mathrm{FFA}(\mathrm{mmol} / \mathrm{l})^{\mathrm{a}}$ & 0.43 & \pm & 0.24 & 0.40 & \pm & 0.24 & 0.46 & \pm & 0.29 & 0.49 & \pm & 0.27 & 0.07 & 0.17 \\
\hline Glycerol $(\mathrm{mmol} /)^{2}{ }^{a}$ & 0.06 & \pm & 0.07 & 0.06 & \pm & 0.03 & 0.10 & \pm & 0.38 & 0.07 & \pm & 0.06 & 0.37 & 0.39 \\
\hline \multicolumn{15}{|l|}{ Women } \\
\hline Genotype & $13 / 13$ & & & $13 / 14$ & & & $13 / 15$ & & & $13 / 16$ & & & $\mathrm{p}$ & $p^{* *}$ \\
\hline$n$ & 438 & & & 31 & & & 282 & & & 73 & & & & \\
\hline Age (years) & 50.1 & \pm & 12.4 & 46.5 & \pm & 12.3 & 51.4 & \pm & 12.7 & 50.9 & \pm & 13.0 & 0.16 & \\
\hline $\mathrm{CT}(\mathrm{mmol} / \mathrm{l})$ & 5.99 & \pm & 1.63 & 5.82 & \pm & 2.27 & 6.02 & \pm & 1.76 & 6.31 & \pm & 1.83 & 0.45 & 0.49 \\
\hline $\mathrm{HDL}-\mathrm{C}(\mathrm{mmol} / \mathrm{l})$ & 1.37 & \pm & 0.48 & 1.31 & \pm & 0.44 & 1.38 & \pm & 0.53 & 1.34 & \pm & 0.51 & 0.83 & 0.88 \\
\hline $\mathrm{LDL}(\mathrm{mmol} / \mathrm{l})$ & 3.57 & \pm & 1.48 & 3.30 & \pm & 0.96 & 3.44 & \pm & 1.17 & 3.67 & \pm & 1.50 & 0.37 & 0.37 \\
\hline $\mathrm{TG}(\mathrm{mmol} / \mathrm{l})^{\mathrm{a}}$ & 1.69 & \pm & 2.11 & 1.68 & \pm & 2.23 & 1.80 & \pm & 2.12 & 1.88 & \pm & 2.15 & 0.49 & 0.63 \\
\hline WG (cm) & 83.5 & \pm & 12.6 & 86.7 & \pm & 13.9 & 85.0 & \pm & 12.4 & 87.0 & \pm & 13.2 & 0.07 & 0.05 \\
\hline BMI $\left(\mathrm{kg} / \mathrm{m}^{2}\right)$ & 26.1 & \pm & 4.9 & 27.9 & \pm & 6.0 & 26.5 & \pm & 4.8 & 27.7 & \pm & 5.1 & 0.019 & 0.013 \\
\hline Glycaemia $(\mathrm{mmol} / \mathrm{l})^{a}$ & 5.37 & \pm & 1.28 & 5.34 & \pm & 0.91 & 5.60 & \pm & 1.87 & 5.74 & \pm & 1.86 & 0.13 & 0.16 \\
\hline Systolic BP (mmHg) & 124.1 & \pm & 21.7 & 122.6 & \pm & 13.4 & 129.7 & \pm & $22.0 \mathrm{a}$ & 135.8 & \pm & $26.5 \mathrm{a}$ & $<0.001$ & $<0.001$ \\
\hline Diastolic BP (mmHg) & 76.5 & \pm & 11.6 & 78.7 & \pm & 10.5 & 78.9 & \pm & 11.8 & 81.3 & \pm & $12.2 \mathrm{a}$ & 0.003 & 0.003 \\
\hline $\mathrm{FFA}(\mathrm{mmol} / \mathrm{l})^{\mathrm{a}}$ & 0.57 & \pm & 0.26 & 0.53 & \pm & 0.23 & 0.55 & \pm & 0.36 & 0.60 & \pm & 0.29 & 0.42 & 0.66 \\
\hline
\end{tabular}


Table 1 Subjects' characteristics according to the SST poly T repeat polymorphism genotype in the entire sample and according to gender (Continued)

\begin{tabular}{|c|c|c|c|c|c|c|c|c|c|c|c|c|c|c|}
\hline $\begin{array}{l}\text { All subjects } \\
\text { Genotype }\end{array}$ & $13 / 13$ & & & $13 / 14$ & & & $13 / 15$ & & & $13 / 16$ & & & $p$ & $\mathrm{p}^{*}$ \\
\hline Glycerol $(\mathrm{mmol} / \mathrm{l})^{a}$ & 0.09 & \pm & 0.11 & 0.08 & \pm & 0.03 & 0.08 & \pm & 0.08 & 0.09 & \pm & 0.05 & 0.28 & 0.13 \\
\hline
\end{tabular}

All data are mean \pm Standard deviation

Significantly different $(p<0.05)$ as compared to (a) $13 / 13$; (b) to $13 / 14$; (c) to $13 / 15$

Significant $p$-value are in bold

$C T$ cholesterol total, $H D L-C$ high density lipoprotein-cholesterol, $L D L-C$ light density lipoprotein-cholesterol, $T G$ triglycerides, WG Waist girth, $B P$ blood pressure, $B M I$ body mass index, FFA free fatty acid

*age and gender included as a covariates

**age included as a covariate

a $=$ geometrical mean and $p$-value obtained after $\log _{10}$ transformation

latter have been found. However, those genetic factors can explain only a small fraction of the population variance. The full spectrum of contributing loci could be larger. The small part of each gene in the expression of this complex trait could result from the interaction between various genes, in addition to an interaction between genes and environmental factors [18].
SST is known to be associated with a variety of physiologic functions, among which many are involved in the expression of metabolic syndrome, including glucose metabolism [19]. However, according to our results, the differential expression of metabolic syndrome according to poly-T repeat length appears mainly due to its association with high blood pressure, dyslipidemia and obesity,

Table 2 Prevalence (\%) of metabolic syndrome components according to the SST poly-T repeat polymorphism genotype

\begin{tabular}{|c|c|c|c|c|c|}
\hline \multirow[b]{2}{*}{ All subjects } & \multicolumn{5}{|c|}{ Genotypes } \\
\hline & $13 / 13$ & $13 / 14$ & $13 / 15$ & $13 / 16$ & $p$ \\
\hline$n$ & 913 & 69 & 571 & 172 & \\
\hline Abdominal Obesity & 58.3 & 65.2 & 59.5 & 69.8 & 0.032 \\
\hline Hypertriglyceridemia & 50.4 & 55.1 & 55.7 & 61.6 & 0.026 \\
\hline Low HDL-C level & 51.2 & 50.7 & 51.5 & 62.2 & 0.058 \\
\hline Hypertension & 33.6 & 23.2 & 39.2 & 42.4 & 0.005 \\
\hline Hyperglycaemia & 36.5 & 46.4 & 38.2 & 45.9 & 0.060 \\
\hline Diagnosis of Metabolic Syndrome & 46.8 & 47.8 & 49.6 & 61.6 & 0.005 \\
\hline \multicolumn{6}{|l|}{ Men } \\
\hline$n$ & 475 & 38 & 289 & 99 & \\
\hline Abdominal Obesity & 74.5 & 73.7 & 72.3 & 80.8 & NS \\
\hline Hypertriglyceridemia & 53.3 & 63.2 & 60.2 & 68.7 & 0.019 \\
\hline Low HDL-C level & 55.6 & 50.0 & 56.4 & 67.7 & NS \\
\hline Hypertension & 33.3 & 21.1 & 34.6 & 35.4 & NS \\
\hline Hyperglycaemia & 43.6 & 55.3 & 42.9 & 49.5 & NS \\
\hline Diagnosis of Metabolic Syndrome & 57.1 & 52.6 & 56.7 & 66.7 & NS \\
\hline \multicolumn{6}{|l|}{ Women } \\
\hline$n$ & 438 & 31 & 282 & 73 & \\
\hline Abdominal Obesity & 40.6 & 54.8 & 46.5 & 54.8 & 0.056 \\
\hline Hypertriglyceridemia & 47.3 & 45.2 & 51.1 & 52.1 & NS \\
\hline Low HDL-C level & 46.3 & 51.6 & 46.5 & 54.8 & NS \\
\hline Hypertension & 34.0 & 25.8 & 44.0 & 52.1 & 0.002 \\
\hline Hyperglycaemia & 28.8 & 35.5 & 33.3 & 41.1 & NS \\
\hline Diagnosis of Metabolic Syndrome & 35.6 & 41.9 & 42.2 & 54.8 & 0.013 \\
\hline
\end{tabular}

NS: $p \geq 0.1$ Abdominal obesity = waist circumference $>102 \mathrm{~cm}$ in men or $>88 \mathrm{~cm}$ in women; Hypertriglyceridemia $=\mathrm{TG}>1.7 \mathrm{mmol} / \mathrm{L} ;$ Low HDL-C level $=\mathrm{HDL}-$ Cholesterol $<1.03 \mathrm{mmol} / \mathrm{L}$ in men or $<1.3 \mathrm{mmol} / \mathrm{L}$ in women; Hypertension = blood pressure $\geq 130 \mathrm{mmHg}$ systolic blood pressure or $\geq 85 \mathrm{mmHg}$ diastolic blood pressure or hypertensive treatment; Hyperglycaemia $=$ fasting glucose $>5.6 \mathrm{mmol} / \mathrm{L}$ or drug treatment for elevated blood glucose Significant $p$-value are in bold 
Table 3 Estimated relative risk (odds ratio) of exhibiting metabolic syndrome components associated with the SST poly-T repeat polymorphism genotype

\begin{tabular}{|c|c|c|c|c|c|c|c|c|}
\hline & Genotypes & & $\begin{array}{l}\text { Abdominal } \\
\text { Obesity }\end{array}$ & Hypertriglyceridemia & Low HDL-Cholesterol & Hypertension & Hyperglycaemia & $\begin{array}{l}\text { Metabolic } \\
\text { Syndrome }\end{array}$ \\
\hline \multirow[t]{7}{*}{ Entire sample ${ }^{a}$} & $13 / 13$ & $\begin{array}{l}p= \\
O R\end{array}$ & 1 & 1 & 1 & 1 & 1 & 1 \\
\hline & $13 / 14$ & OR & 1.46 & 1.24 & 0.96 & 0.66 & 1.68 & 1.10 \\
\hline & & $\begin{array}{l}p \text {-value } \\
95 \% \mathrm{Cl}\end{array}$ & $\begin{array}{l}0.18 \\
(0.84-2.53)\end{array}$ & $\begin{array}{l}0.39 \\
(0.76-2.04)\end{array}$ & $\begin{array}{l}0.87 \\
(0.59-1.57)\end{array}$ & $\begin{array}{l}0.18 \\
(0.36-1.21)\end{array}$ & $\begin{array}{l}0.05 \\
(1.01-2.81)\end{array}$ & $\begin{array}{l}0.71 \\
(0.66-1.83)\end{array}$ \\
\hline & $13 / 15$ & OR & 1.07 & 1.24 & 1.02 & 1.26 & 1.07 & 1.13 \\
\hline & & $\begin{array}{l}\text { p-value } \\
95 \% \mathrm{Cl}\end{array}$ & $\begin{array}{l}0.57 \\
(0.85-1.34)\end{array}$ & $\begin{array}{l}0.05 \\
(1.01-1.53)\end{array}$ & $\begin{array}{l}0.85 \\
(0.83-1.26)\end{array}$ & $\begin{array}{l}0.05 \\
(1.00-1.59)\end{array}$ & $\begin{array}{l}0.53 \\
(0.86-1.34)\end{array}$ & $\begin{array}{l}0.28 \\
(0.91-1.40)\end{array}$ \\
\hline & $13 / 16$ & OR & 1.60 & 1.55 & 1.55 & 1.48 & 1.43 & 1.79 \\
\hline & & $\begin{array}{l}\text { p-value } \\
95 \% \mathrm{Cl}\end{array}$ & $\begin{array}{l}0.01 \\
(1.10-2.33)\end{array}$ & $\begin{array}{l}0.01 \\
(1.11-2.17)\end{array}$ & $\begin{array}{l}0.01 \\
(1.11-2.17)\end{array}$ & $\begin{array}{l}0.03 \\
(1.04-2.11)\end{array}$ & $\begin{array}{l}0.04 \\
(1.02-2.02)\end{array}$ & $\begin{array}{l}<0.01 \\
(1.27-2.53)\end{array}$ \\
\hline \multirow[t]{7}{*}{ Men $^{\mathrm{b}}$} & $13 / 13$ & OR & 1 & 1 & 1 & 1 & 1 & 1 \\
\hline & $13 / 14$ & OR & 0.99 & 1.49 & 0.79 & 0.55 & 1.71 & 0.85 \\
\hline & & $\begin{array}{l}\text { p-value } \\
95 \% \mathrm{Cl}\end{array}$ & $\begin{array}{l}0.98 \\
(0.46-2.12)\end{array}$ & $\begin{array}{l}0.25 \\
(0.75-2.96)\end{array}$ & $\begin{array}{l}0.50 \\
(0.41-1.54)\end{array}$ & $\begin{array}{l}0.15 \\
(0.24-1.25)\end{array}$ & $\begin{array}{l}0.12 \\
(0.87-3.37)\end{array}$ & $\begin{array}{l}0.64 \\
(0.66-1.83)\end{array}$ \\
\hline & $13 / 15$ & OR & 0.91 & 1.32 & 1.03 & 1.09 & 0.99 & 1.00 \\
\hline & & $\begin{array}{l}\text { p-value } \\
95 \% \mathrm{Cl}\end{array}$ & $\begin{array}{l}0.58 \\
(0.65-1.27)\end{array}$ & $\begin{array}{l}0.07 \\
(0.98-1.78)\end{array}$ & $\begin{array}{l}0.84 \\
(0.77-1.39)\end{array}$ & $\begin{array}{l}0.59 \\
(0.79-1.50)\end{array}$ & $\begin{array}{l}0.96 \\
(0.73-1.34)\end{array}$ & $\begin{array}{l}1.00 \\
(0.91-1.40)\end{array}$ \\
\hline & $13 / 16$ & OR & 1.43 & 1.93 & 1.68 & 1.08 & 1.26 & 1.50 \\
\hline & & $\begin{array}{l}\text { p-value } \\
95 \% \mathrm{Cl}\end{array}$ & $\begin{array}{l}0.20 \\
(0.83-2.47)\end{array}$ & $\begin{array}{l}0.01 \\
(1.22-3.07)\end{array}$ & $\begin{array}{l}0.03 \\
(1.06-2.65)\end{array}$ & $\begin{array}{l}0.75 \\
(0.68-1.72)\end{array}$ & $\begin{array}{l}0.31 \\
(0.81-1.96)\end{array}$ & $\begin{array}{l}0.08 \\
(0.95-2.37)\end{array}$ \\
\hline \multirow[t]{7}{*}{ Women $^{\mathrm{b}}$} & $13 / 13$ & OR & 1 & 1 & 1 & 1 & 1 & 1 \\
\hline & $13 / 14$ & OR & 2.08 & 1.04 & 1.21 & 0.86 & 1.66 & 1.59 \\
\hline & & $\begin{array}{l}\text { p-value } \\
95 \% \mathrm{Cl}\end{array}$ & $\begin{array}{l}0.06 \\
(0.98-4.42)\end{array}$ & $\begin{array}{l}0.91 \\
(0.49-2.21)\end{array}$ & $\begin{array}{l}0.61 \\
(0.58-2.52)\end{array}$ & $\begin{array}{l}0.74 \\
(0.35-2.11)\end{array}$ & $\begin{array}{l}0.21 \\
(0.75-3.67)\end{array}$ & $\begin{array}{l}0.24 \\
(0.74-3.43)\end{array}$ \\
\hline & $13 / 15$ & OR & 1.22 & 1.12 & 1.01 & 1.45 & 1.17 & 1.26 \\
\hline & & $\begin{array}{l}\text { p-value } \\
95 \% \mathrm{Cl}\end{array}$ & $\begin{array}{l}0.21 \\
(0.89-1.66)\end{array}$ & $\begin{array}{l}0.49 \\
(0.82-1.52)\end{array}$ & $\begin{array}{l}0.94 \\
(0.75-1.37)\end{array}$ & $\begin{array}{l}0.03 \\
(1.03-2.04)\end{array}$ & $\begin{array}{l}0.35 \\
(0.84-1.64)\end{array}$ & $\begin{array}{l}0.16 \\
(0.91-1.73)\end{array}$ \\
\hline & $13 / 16$ & OR & 1.77 & 1.19 & 1.41 & 2.33 & 1.73 & 2.25 \\
\hline & & $\begin{array}{l}\text { p-value } \\
95 \% \mathrm{Cl}\end{array}$ & $\begin{array}{l}0.03 \\
(1.06-2.95)\end{array}$ & $\begin{array}{l}0.51 \\
(0.72-1.98)\end{array}$ & $\begin{array}{l}0.18 \\
(0.86-2.32)\end{array}$ & $\begin{array}{l}<0.01 \\
(1.33-4.09)\end{array}$ & $\begin{array}{l}0.04 \\
(1.02-2.93)\end{array}$ & $\begin{array}{l}<0.01 \\
(1.33-3.79)\end{array}$ \\
\hline
\end{tabular}

${ }^{a}$ Including age and sex as covariates

${ }^{b}$ Including age as a covariate

Significant $p$-values were italicized

while glycaemia homeostasis does not appear to be significantly affected.

As previously published, the SST poly-T repeat polymorphism is significantly associated with increased blood pressure and risk of hypertension, particularly in women and in overweight/obese individuals. Various pathways could explain this association. Among them, inhibition of the vasodilator glucagon, a decrease in plasma renin activity, facilitation of vasoconstrictors, or the relationship with neuronostatin may be involved in the rise of blood pressure [6]

Effects of SST on lipid levels have been only partially investigated and published conclusions contain apparent discrepancies. SST analogues administered in the context of acromegaly seem to inhibit GH release, with favourable changes in plasma lipids, and improvement of insulin sensitivity [20]. However, increased fat mass, an increase in TG level and reduced HDL-cholesterol levels have been observed in untreated GH deficiency. Moreover, some observations have shown that GH level is negatively associated with adipose tissue mass, while GH replacement has been shown to improve those factors in dose-dependant levels [21]. GH deficiency and acromegaly are at the opposite ends of the GH level spectrum. In acromegaly, the improvement of lipid profile with SST analogues might be the result of a reduction in GH secretion. However, in a non-acromegalic individual, increased SST output may suppress GH secretion and, as a result, may be the cause of GH deficiency. Thus, it can be hypothesized that increased SST secretion may cause hypertriglyceridemia and a low level of HDL-cholesterol [21]. 
Because the prevalence of abdominal obesity appears to rise with an increase of the poly- $T$ repeat length, we cannot exclude the possibility that the obesity could, at least partially, cause the observed hypertriglyceridemia and low HDL-cholesterol levels. Although there are several reports showing that the administration of SST plays a role in eating behaviours and body weight, the results have been controversial, probably because of the multiple actions of this peptide on central and gastrointestinal functions [22]. Accordingly, sizeable ingestion of macronutrients, including carbohydrates, fat and protein can induce low-grade inflammation and reduced secretion of intestinal SST. These effects may in turn increase intestinal absorption of energy and nutrients and the formation of adipose tissues. The SST analogue octreotide has been shown to reverse this process in order to regulate the energy balance. SST may also act on obesity by limiting insulin release, which is the primary hormonal mediator of adipogenesis in humans [8]. Conversely, it has been reported that SST may modulate leptin signalling in hypothalamus and ghrelin circulation and then serves as an orexigenic neuropeptide [23]. Injections of SST agonist over long periods have reduced body weight gain and lean mass independently of food intake in lean rats. These effects are related to $\mathrm{GH}$ inhibition. However, the same SST agonist has stimulated increased food intake and fat mass in diet-induced obese rats. Indeed, several studies have indicated that obesity is associated with enhanced SST quantity and responsiveness and that inhibition of hypothalamic SST release consistently improves GH response in obese subjects [24]. Moreover, polymorphisms in the SST gene receptor have been associated with anthropometric variables and increased food intake. The relationship between SST and obesity does not appear to be simple, and recent evidence points to a divergent action of SST in the brain and in the periphery (gut, stomach, pancreas) [24].

These observations agree with our results. Indeed, mean concentrations of almost all the components studied increase from genotype $13 / 13$ to $13 / 16$. Although they are not all significant, these associations may contribute to the increase in metabolic syndrome elements, according to genotype. The features of metabolic syndrome are interrelated and share common pathways, and it is still not fully understood how these components interact over time, and what the real impact of genetics and the environment is on each of them. Thus, the effects of SST may be far more complex than initially expected, but their study may help to build a better understanding of the interplay among metabolic syndrome components.

We observed differences between men and women. SST regulates $\mathrm{GH}$ release and synthesis in a pulsatile pattern that is gender dependent. GH deficiency is associated with increased fat mass and lower lean body mass, metabolic derangements, including insulin resistance, and suboptimal physical performance [25]. Under a normal fed condition, $\mathrm{GH}$ release in males tends to be highly organized, but it is disorganized in females [26]. In the obese state, SST levels are elevated in the portal circulation, and systemic immunoneutralization of SST restores larger $\mathrm{GH}$ pulses. Thus, our current observations are in accordance with other studies, which demonstrate that SST output is more critical in females than males, but may be relevant for both of them in the case of abdominal obesity [27]. However, detailed studies examining the gender-dependent role SST plays in regulating $\mathrm{GH}$ pulse release in humans has not been directly studied and therefore caution should be exercised in extrapolating the results of experiments across species. Another possible explanation for these differences could be related to fat distribution. Usually, men present with more abdominal fat than women and thus, for a given excess of BMI, women may be less prone to developing other metabolic alterations [28]. This may explain that, while the means of WG and BMI are significant in women, the other components are not. However, we cannot exclude the possibility that those differences may also be influenced by other hormonal or environmental factors.

In this study, only slight phenotypic changes were observed, as in strains of knockout mouse created in order to study SST function [29]. Those results suggest that the poly-T repeat polymorphisms in the SST promoter region may influence several processes involved in metabolic syndrome, probably by acting as a fine-tuning regulator of energy metabolism. Even if the contribution to the variation differs between genders, it seems to follow the same trend. The hypothesis that an increase in the number of $\mathrm{T}$ repeats could act by increasing SST output is appealing, but more research is needed to better understand the processes that determine the genetic regulation of SST on metabolic syndrome components and particularly, to clarify its specific role.

The French-Canadian population of Saguenay-Lac-St-Jean, from which our subjects originate, is a strength in our study. This population descended from a founder population that settled in this region 300-400 years ago. This founder effect provides several benefits for mapping the genomic determinants of complex traits [30]. Genetic heterogeneity remains a problem in disease identification strategies that can be avoided by analyzing homogeneous populations with geographic stability, and which are most likely uniform in their environmental exposure [31].

Our study has however some limitations. All groups have an average BMI and waist circumference over the limit of overweight, except for waist girth among women with 13/13 genotypes. This could act as confounder because, as previously seen, abdominal obesity is well 
known to disrupt SST quantity and responsiveness. Another limitation is selection bias, because participants were recruited among lipid clinic patients. This may have led to a higher prevalence of alteration in lipid profile or components such as T2D. Moreover, we cannot exclude the possibility that other genetic polymorphisms may influence lipid profile and the results. Thus, it is essential to reproduce these analyses in other populations with different phenotypes.

\section{Conclusions}

Our study suggests that the polymorphism in the SST gene promoter is associated with the onset of metabolic syndrome. Our results vary along gender lines but the same tendency seems to be found in both sexes. However, as noted, this study was performed among subjects issued from studies on genetic determinants of T2D and/or CAD, so that the proportion of subjects with normal metabolic profile is quite under what is observed in the general population. Obtained results therefore need to be confirmed among other samples, more representative of the general population.

\section{Abbreviations}

ANCOVA: Analyse of covariance; ANOVA: Analyse of Variance; CAD: Coronary artery diseases; GH: Growth Hormone; GWAS: Genome-wide Association Studies; HDL: High density lipoprotein; SST: Somatostatin; T2D: Type 2 diabetes mellitus; TG: Triglycerides; X2: Chi square

\section{Acknowledgements}

The authors thank all the staff of the ECOGENE-21 Clinical Research Center and Chicoutimi Hospital Lipid Clinic for their help in data collection and their dedicated work. Finally, we would like to acknowledge the contribution of the subjects who participated in this project; without them, studies would not be possible.

\section{Funding}

This research was supported by ECOGENE-21, the CIHR team in community genetics (grant \#CTP-82941).

\section{Availability of data and materials}

The datasets used and analyzed during the current study are available from the corresponding author on reasonable request.

\section{Authors' contributions}

MT has conceived the study design, performed the data analysis/ interpretation and written the manuscript. DB and DG have conceived the study design and revised the manuscript. All authors have approved the manuscript's submission.

\section{Ethics approval and consent to participate}

This project received the approval of the Chicoutimi Hospital Ethics Committee \# 2007-009 et 2007-010 and was conducted in accordance with the Declaration of Helsinki. All subjects gave their written informed consent to participate in this study.

\section{Consent for publication}

Not applicable.

\section{Competing interests}

The authors declare that they have no competing interests.

\section{Publisher's Note}

Springer Nature remains neutral with regard to jurisdictional claims in published maps and institutional affiliations.

Received: 13 March 2018 Accepted: 4 July 2018

Published online: 27 July 2018

\section{References}

1. Alberti KG, Eckel RH, Grundy SM, Zimmet PZ, Cleeman Jl, Donato KA, et al. Harmonizing the metabolic syndrome: a joint interim statement of the international diabetes federation task force on epidemiology and prevention; National Heart, Lung, and Blood Institute; American Heart Association; world heart federation; international atherosclerosis society; and International Association for the Study of obesity. Circulation 2009;120: 1640-1645.

2. Aguilar M, Bhuket T, Torres S, Liu B, Wong RJ. Prevalence of the metabolic syndrome in the United States, 2003-2012. JAMA. 2015;313:1973-4.

3. Berndt SI, Gustafsson S, Magi R, Ganna A, Wheeler E, Feitosa MF, et al. Genome-wide meta-analysis identifies 11 new loci for anthropometric traits and provides insights into genetic architecture. Nat Genet 2013:45:501-512.

4. Morris AP, Voight BF, Teslovich TM, Ferreira T, Segre AV, Steinthorsdottir V, et al. Large-scale association analysis provides insights into the genetic architecture and pathophysiology of type 2 diabetes. Nat Genet 2012;44: 981-990.

5. Manolio TA. Bringing genome-wide association findings into clinical use. Nat Rev Genet. 2013;14:549-58.

6. Tremblay M, Brisson D, Gaudet D. Association between a polymorphic poly$T$ repeat sequence in the promoter of the somatostatin gene and hypertension. Hypertens Res. 2016;39:467-74.

7. Hauge-Evans AC, King AJ, Carmignac D, Richardson CC, Robinson IC, Low $\mathrm{MJ}$, et al. Somatostatin secreted by islet delta-cells fulfills multiple roles as a paracrine regulator of islet function. Diabetes 2009;58:403-411.

8. Corleto VD. Somatostatin and the gastrointestinal tract. Curr Opin Endocrinol Diabetes Obes. 2010;17:63-8.

9. Li W, Shi YH, Yang RL, Cui J, Xiao Y, Wang B, et al. Effect of somatostatin analog on high-fat diet-induced metabolic syndrome: involvement of reactive oxygen species. Peptides 2010:31:625-629.

10. Gaudet D, Arsenault S, Perusse L, Vohl MC, St-Pierre J, Bergeron J, et al. Glycerol as a correlate of impaired glucose tolerance: dissection of a complex system by use of a simple genetic trait. Am J Hum Genet 2000;66: 1558-1568.

11. Vohl MC, Lepage P, Gaudet D, Brewer CG, Betard C, Perron P, et al. Molecular scanning of the human PPARa gene: association of the L162V mutation with hyperapobetalipoproteinemia. J Lipid Res 2000;41:945-952

12. The Airlie (VA) Consensus Conference.Standardization of Anthropometric Measurements In: Human Kinetics: . EDN Edited by Lohman T RA, Martorel R, eds. Champaign,IL; 1988. p 39-80.

13. Gaudet D, Arsenault S, Belanger C, Hudson T, Perron P, Bernard M, et al. Procedure to protect confidentiality of familial data in community genetics and genomic research. Clin Genet 1999;55:259-264.

14. Havel RJ, Eder HA, Bragdon JH. The distribution and chemical composition of ultracentrifugally separated lipoproteins in human serum. J Clin Invest. 1955:34:1345-53.

15. McNamara JR, Schaefer EJ. Automated enzymatic standardized lipid analyses for plasma and lipoprotein fractions. Clin Chim Acta. 1987;166:1-8.

16. Friedewald WT, Levy RI, Fredrickson DS. Estimation of the concentration of low-density lipoprotein cholesterol in plasma, without use of the preparative ultracentrifuge. Clin Chem. 1972;18:499-502.

17. Chen $Y$, Zhang $X$, Pan B, Jin X, Yao H, Chen B, et al. A modified formula for calculating low-density lipoprotein cholesterol values. Lipids Health Dis 2010;9:52

18. Zhang Y, Kent JW, Jr., Olivier M, Ali O, Broeckel U, Abdou RM, et al. QTLbased association analyses reveal novel genes influencing pleiotropy of metabolic syndrome (MetS). Obesity (Silver Spring) 2013;21:2099-2111.

19. Verges B. Effects of anti-somatostatin agents on glucose metabolism. Diabetes Metab. 2017:43:411-5.

20. Dal J, List EO, Jorgensen JO, Berryman DE. Glucose and fat metabolism in acromegaly: from mice models to patient care. Neuroendocrinology. 2016; 103:96-105

21. Vijayakumar A, Yakar S, Leroith D. The intricate role of growth hormone in metabolism. Front Endocrinol. 2011;2:32. 
22. Rai U, Thrimawithana TR, Valery C, Young SA. Therapeutic uses of somatostatin and its analogues: current view and potential applications. Pharmacol Ther. 2015;152:98-110.

23. Stepanyan Z, Kocharyan A, Behrens M, Koebnick C, Pyrski M, Meyerhof W. Somatostatin, a negative-regulator of central leptin action in the rat hypothalamus. J Neurochem. 2007;100:468-78,

24. Stengel A, Coskun T, Goebel-Stengel M, Craft LS, Alsina-Fernandez J, Wang $\mathrm{L}$, et al. Chronic injection of pansomatostatin agonist ODT8-SST differentially modulates food intake and decreases body weight gain in lean and dietinduced obese rats. Regul Pept 2011;167:201-208.

25. Popovic V. Approach to testing growth hormone $(\mathrm{GH})$ secretion in obese subjects. J Clin Endocrinol Metab. 2013;98:1789-96.

26. Cordoba-Chacon J, Gahete MD, Castano JP, Kineman RD, Luque RM. Somatostatin and its receptors contribute in a tissue-specific manner to the sex-dependent metabolic (fed/fasting) control of growth hormone axis in mice. Am J Physiol Endocrinol Metab. 2011;300:E46-54.

27. Steyn FJ, Tolle V, Chen C, Epelbaum J. Neuroendocrine regulation of growth hormone secretion. Compr Physiol. 2016;6:687-735.

28. Tchernof A, Despres JP. Pathophysiology of human visceral obesity: an update. Physiol Rev. 2013;93:359-404.

29. Low MJ. Clinical endocrinology and metabolism. The somatostatin neuroendocrine system: physiology and clinical relevance in gastrointestinal and pancreatic disorders. Best Pract Res Clin Endocrinol Metab. 2004;18: $607-22$

30. Engert JC, Lemire M, Faith J, Brisson D, Fujiwara TM, Roslin NM, et al. Identification of a chromosome $8 p$ locus for early-onset coronary heart disease in a French Canadian population. Eur J Hum Genet 2008;16:105114.

31. Qi L, Menzaghi C, Salvemini L, De Bonis C, Trischitta V, Hu FB. Novel locus FER is associated with serum HMW adiponectin levels. Diabetes. 2011;60: 2197-201.

Ready to submit your research? Choose BMC and benefit from:

- fast, convenient online submission

- thorough peer review by experienced researchers in your field

- rapid publication on acceptance

- support for research data, including large and complex data types

- gold Open Access which fosters wider collaboration and increased citations

- maximum visibility for your research: over $100 \mathrm{M}$ website views per year

At $\mathrm{BMC}$, research is always in progress.

Learn more biomedcentral.com/submissions 